\title{
Editorial
}

\section{Advanced Communication Models and Services for Smart World}

\author{
James J. Park, ${ }^{1}$ Ning Zhang, ${ }^{2}$ Neil Y. Yen, ${ }^{3}$ and Muhammad K. Khan ${ }^{4}$ \\ ${ }^{1}$ Seoul National University of Science and Technology, 172 Gongreung 2-dong, Nowon-gu, Seoul 139-743, Republic of Korea \\ ${ }^{2}$ University of Manchester, 2.113 Kilburn Building, Oxford Road, Manchester M13 9PL, UK \\ ${ }^{3}$ University of Aizu, Tsuruga, Ikkimachi, Aizu-Wakamatsu, Fukushima 9658580, Japan \\ ${ }^{4}$ King Saud University, P.O. Box 92144, Riyadh 11653, Saudi Arabia \\ Correspondence should be addressed to James J. Park; jamespark.seoul@gmail.com
}

Received 8 April 2015; Accepted 8 April 2015

Copyright (C) 2015 James J. Park et al. This is an open access article distributed under the Creative Commons Attribution License, which permits unrestricted use, distribution, and reproduction in any medium, provided the original work is properly cited.

Smart world (SW) represents a future communication environment featuring daily-life objects that continuously involve nonexpert users. The SW has rapidly emerged as an exciting new paradigm which integrates different active research areas such as Internet of Things (IoT), ubiquitous and pervasive computing, and peer-to-peer communications providing new exciting seamless services for the future IT environments. The systems for SW manage lots of sensors and mobile devices which continuously update information from the real-world objects, and most data are generated automatically through intelligent communication environments. This special issue aims to address advanced communication models and services for SW. It will solicit original research papers on topics including effective IoT, ubiquitous and pervasive computing, context-based services, machine-to-machine service model integration of legacy systems and services, security, business models, and novel applications of SW utilization.

During the working period, we received 20 submissions from at least 10 different countries where the corresponding authors were majorly counted by the deadline for manuscript submission. All these submissions were considered significant in the area of the promising services for the development of smart world, but, however, only two-fifth of them passed the first-round examination which is based on a strict and rigorous review policy. After a two-round review process, only 6 papers were accepted for being included in this issue. These accepted papers mainly look at our issue from the perspectives of smart business, intelligent web, e-commerce, semantic web, security and privacy, digital image processing, social network analysis, user behavior, mobile gateway, ubiquitous platform, mobile cloud, open API, human-computer interaction, emotion detection, wireless sensor network, network optimization, and power grid, which brought lively and focused discussions to the publics.

The paper titled "Getting Business Insights through Clustering Online Behaviors" explores the behaviors of online users. The major concern of this research is to identify clearly the frequent activities since the Internet has played a rather important role in present marketing environment especially to the companies. Authors utilized the revised $K$-means for the analysis of user behavior and attempt to obtain the clickstream data. Four major issues are investigated, which all point out the importance of the wide use of online media such as e-commerce marketing, social network, and online community.

The paper titled "An Intelligent Web Digital Image Metadata Service Platform for Social Curation Commerce Environment" introduces an intelligent web digital image information retrieval platform, which adopts XML technology for social caution commerce environment. To support objectbased content retrieval on product catalog images containing multiple objects, we describe a multilevel metadata structures representing the local features, global features, and semantics of image data. To enable semantic-based and content-based retrieval on such image data, we design an XML-Schema for the proposed metadata. Authors also describe how to automatically transform the retrieval results into the forms suitable for the various user environments, such as web 
browser or mobile device, using XSLT. The proposed scheme can be utilized to enable efficient e-catalog metadata sharing between systems, and it will contribute to improving the retrieval correctness and the user's satisfaction on semanticbased web digital image information retrieval.

The paper titled "Design of Mobile Gateway for Implementation of Smart Work System” considers a novel design of smart work environment. The smart work platform has been designed to meet the diverse needs from public users for efficient process of assigned tasks. For this reason, authors have developed a mobile gateway that is based on the communication server construction OPEN API development, management of mobile ID, protocol design, and design of SSL/TLS security tunnel. Also, authors developed a smart work platform that when you apply this, you are trying to provide information systems environment of mobile company.

The paper titled "Event-Related Potentials Related to Anxiety in Emotion-Attention Interaction" investigates ERP (event-related potential) characteristics using IAPS images, emotional faces, and emotional words as the emotional stimuli presented. The ultimate goal of this study is to detect the ERP components related to state and trait anxiety in an environment which requires target identification by inhibiting task-irrelevant emotional distractors, that is, interaction between emotion and attention. These neural characteristics can help us to understand the effects of anxiety on cognition processing and to determine therapy methods and therapy processes related to anxiety disorder.

The paper titled "Achieving Fair Spectrum Allocation and Reduced Spectrum Handoff in Wireless Sensor Networks: Modeling via Biobjective Optimization" considers the problem of centralized spectrum allocations in wireless sensor networks towards the three main expectations: (1) maximizing fairness, (2) reflecting the priority among sensor data, and (3) avoiding unnecessary spectrum handoff. This problem is studied through a multiobjective mixed integer nonconvex nonlinear programming that is definitely difficult to solve at least globally without any aid of conversion or approximation. To tackle this intractability, authors first convexify the original problem using arithmetic-geometric mean approximation and logarithmic change of the decision variables and then deploy weighted Chebyshev norm-based scalarization method in order to collapse the multiobjective problem into a single objective one. Finally, authors apply simple rounding method in order to obtain approximate integer solutions. The results obtained from the numerical experiments show that, by adjusting the weight on each objective function, the proposed algorithm allocates spectrum bands fairly with well observing each sensor's priority and reduced spectrum handoffs.

The paper titled "Computer Aided Modeling and Analysis of Five-Phase PMBLDC Motor Drive for Low Power High Torque Application" discusses the scenario to achieve high torque at low power with high efficiency. A new five-phase permanent magnet brushless dc (PMBLDC) motor design was analyzed and optimized. A similar three-phase motor having the same $D / L$ ratio (inner diameter $(D)$ and length of the stator $(L))$ is compared for maximum torque and torque ripple of the designed five-phase PMBLDC motor. Maxwell software was used to build finite element simulation model of the motor. The internal complicated magnetic field distribution and dynamic performance simulation were obtained in different positions. No load and load characteristics of the five-phase PMBLDC motor were simulated, and the power consumption of materials was computed. The conformity of the final simulation results indicates that this method can be used to provide a theoretical basis for further optimal design of this new type of motor with its drive, so as to improve the starting torque and reduce torque ripple of the motor.

The scenario of smart world and its related systems, applications, and services has drawn attentions to the publics and indeed caused great changes to our daily lives. With the success in the organization of this special issue, it becomes possible for researchers (and interesting readers as well) who have been engaged in this or related areas to receive state-of-the-art information, gain experiences, and further bring about the benefits in this promising area of study. We, the guest editors, also envision the advanced stimulation of development of innovative services and solutions in this area can be achieved in the coming future.

James J. Park Ning Zhang

Neil Y. Yen

Muhammad K. Khan 

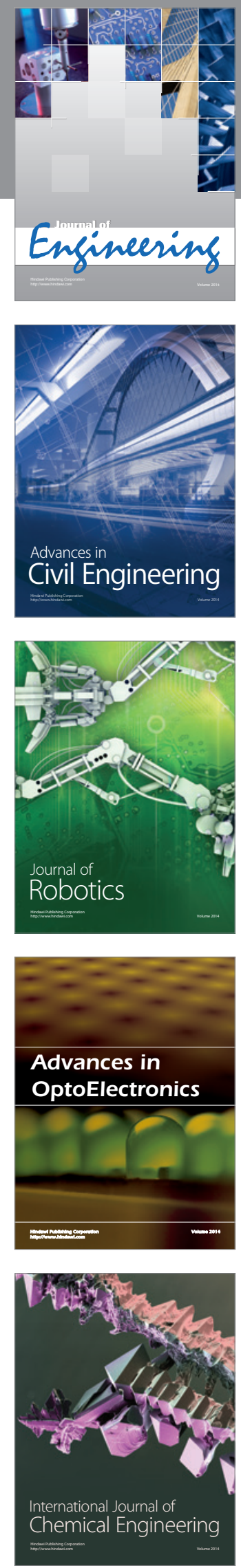

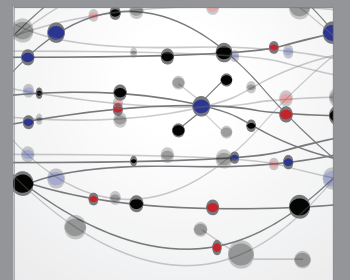

The Scientific World Journal
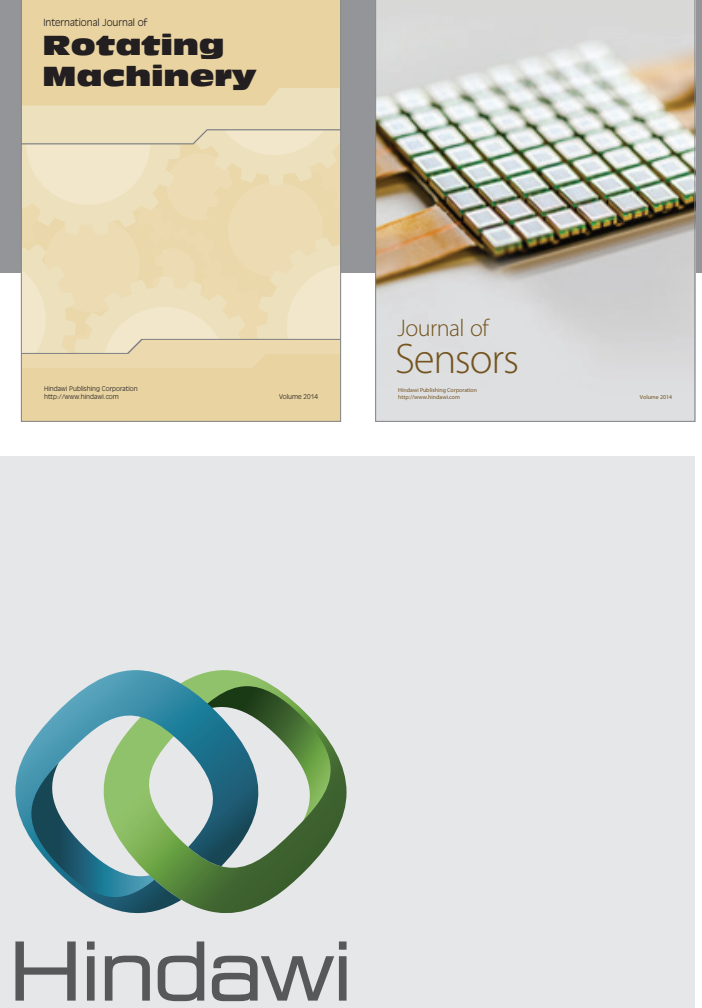

Submit your manuscripts at http://www.hindawi.com
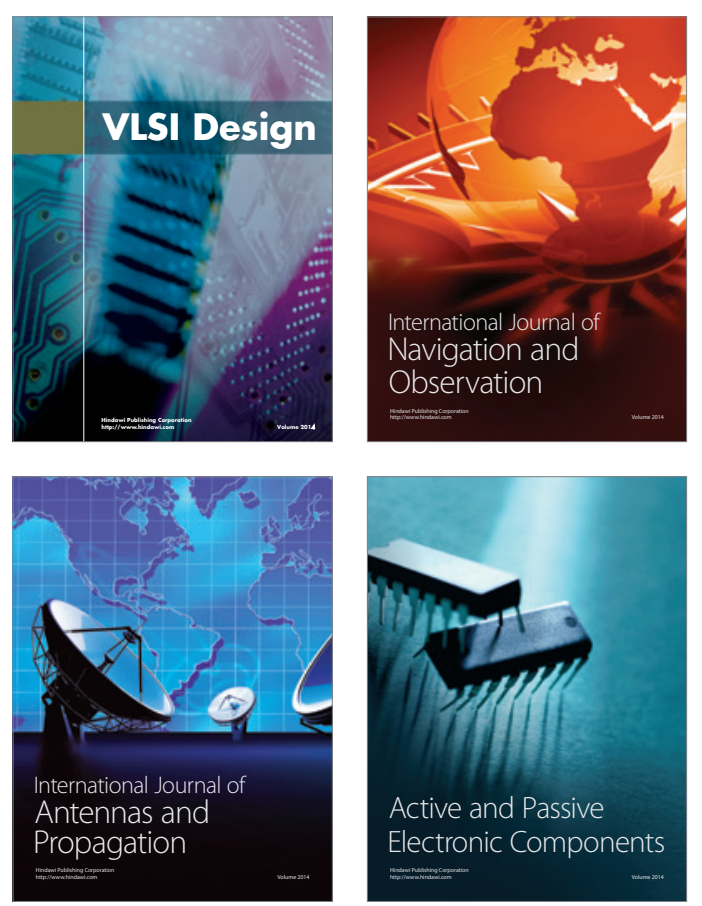
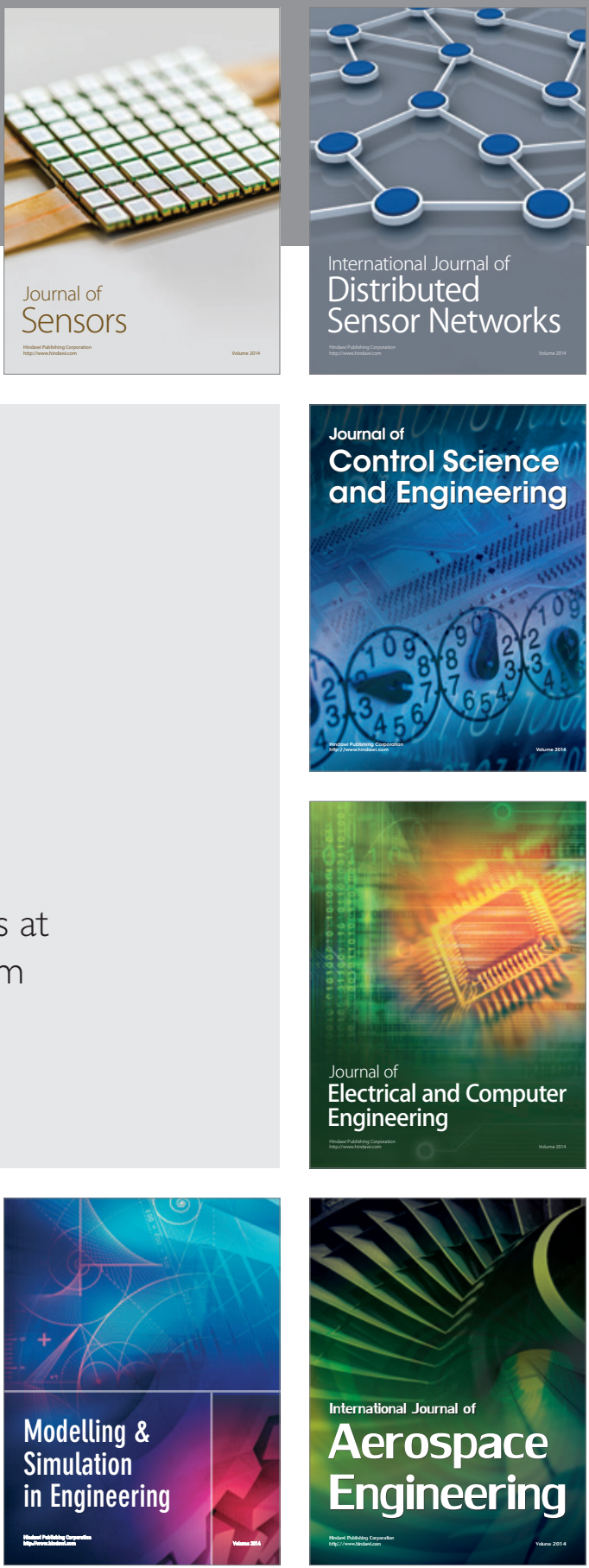

Journal of

Control Science

and Engineering
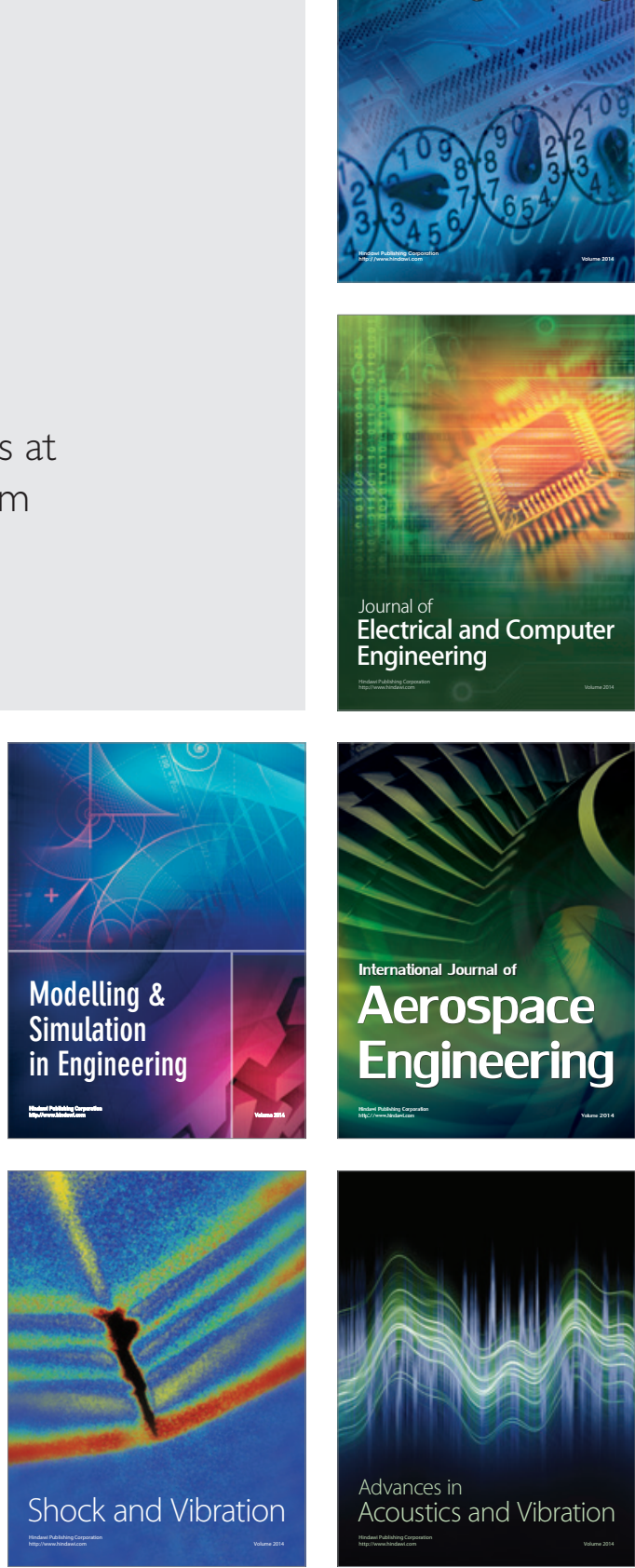